\title{
EDITORIAL
}

\section{Cáncer de mama, cultura de prevención poblacional y profesionalización de su detección y control}

C on motivo del día mundial contra el cáncer de mama la revista Salud Pública de México invitó a un distinguido grupo de investigadores de Estados Unidos, Canadá, Francia, Argentina y México, muchos de ellos amigos y colaboradores del Instituto Nacional de Salud Pública, para presentar las hipótesis de estudio que en el futuro inmediato deberán de responderse para entender la historia natural de esta enfermedad. Esta información pone de relieve el peso del cáncer de mama en el plano mundial a través de las cifras de incidencia y mortalidad que refieren los registros de cáncer internacionales, información que es presentada en la primera contribución de este número. Asimismo, se establece que desde 1980 la mortalidad por este tumor se ha incrementado en México y supera desde 2006 a la mortalidad por cáncer cervicouterino. Las diferencias regionales en nuestro país indican la necesidad de investigación en este campo para recomendar e implantar programas específicos según las necesidades de cada región. El presidente de la Sociedad Americana de Cáncer refiere que el uso de programas de tamizaje organizado mediante mastografía ha mostrado reducir las tasas de mortalidad en países desarrollados; además menciona la importancia de la exploración clínica y la autoexploración en la concientización de la población respecto de la posibilidad de curación de esta enfermedad cuando se diagnostica y trata oportuna y adecuadamente. Se hace énfasis en la necesidad de contar con un programa de control de calidad para que el tamizaje organizado sea eficaz. A este respecto, la norma actual mexicana NOM041-SSA2-2011 cuenta ya con un apartado de control y gestión de calidad y con otro de monitoreo y evaluación del desempeño.
Las diferencias observadas en cuanto a incidencia y mortalidad entre regiones se deben, entre otras razones, a estilos de vida, detección oportuna, diagnóstico y tratamientos diversos. Se incluyen dos trabajos que describen los efectos del consumo de alcohol y dieta sobre el riesgo de cáncer de mama. Se presentan también algunos aspectos teóricos en relación con el proceso de carcinogénesis, indispensable base de conocimiento para explicar el fenómeno del cáncer y guiar las investigaciones sobre este tema.

Se ha argumentado que en la mayoría de los cánceres existe una predisposición y un componente genético. Dos contribuciones de este número discuten estudios genéticos recientes que han identificado nuevas vías de señalización que contribuyen a un mejor conocimiento de la biología de esta enfermedad y a tener así mejor capacidad para prevenirla y tratarla. El cáncer de mama hereditario representa entre 5 y $10 \%$ de todos los casos de cáncer de mama. Aunque el porcentaje no es muy alto, en este número se presentan las características más importantes de las mujeres que enfrentan un riesgo elevado de padecer este tumor y la orientación que debe dárseles en cuanto a medidas de prevención, diagnóstico oportuno, vigilancia y tratamiento específicos.

El cáncer de mama es un desafío en salud pública que enfrentan los servicios de atención primaria, así como de segundo y tercer nivel, no sólo por la magnitud de su incidencia sino también porque las herramientas de tamizaje con las que contamos hoy en día tienen limitaciones en su valor diagnóstico, existen muy bajas coberturas de detección y hay poca profesionalización en los programas de prevención y control. Es necesario el desarrollo de sistemas modernos de vigilancia epide- 
miológica ante la ausencia de registros poblacionales de cáncer en nuestro país. Por esta razón, para enfrentar el cáncer de mama y otros tumores malignos en general, será necesario reactivar la iniciativa de creación del Consejo Nacional Contra el Cáncer, mediante el cual se podrá promover una cultura de prevención en el ámbito poblacional y potenciar los esfuerzos de las instituciones gubernamentales, académicas y de la sociedad civil. Sólo de esta forma se podrá ofrecer una respuesta social organizada para enfrentar este reto y así poder mejorar la calidad de vida de las mujeres.

Agradecemos a todos los investigadores por su amable disposición a participar en este número especial y por la excelente calidad de sus contribuciones.

Dra. Gabriela Torres-Mejía, Dra. Angélica Ángeles-Llerenas, * Dr. Eduardo Lazcano-Ponce.

${ }^{*}$ Centro de Investigación en Salud Poblacional. Instituto Nacional de Salud Pública. 